\section{FRI0018 ASSOCIATION OF MICRORNA-146A RS57095329 POLYMORPHISM WITH SUSCEPTIBILITY TO GOUT TOPHI IN A CHINESE HAN POPULATION}

Yu-Feng Qing ${ }^{1}$, Ting Yi ${ }^{1}$, Zi-Yi Tang ${ }^{1}$, Jian-Xiong Zheng ${ }^{1}$, Qin Xiong ${ }^{1}$, QuanBo Zhang ${ }^{2} .{ }^{1}$ Institute of Rheumatology and Immunology, Affiliated Hospital of North Sichuan Medical College, Department of Rheumatology and Immunology, Affiliated Hospital of North Sichuan Medical College, nanchong, China; ${ }^{2}$ Institute of Rheumatology and Immunology, Affiliated Hospital of North Sichuan Medical College, Department of Geriatrics, affiliated Hospital of North Sichuan Medical College, nanchong, China

Background: MicroRNA-146a (miR-146a) plays an important role in regulation of autoinflammatory diseases including gout[1]. Growing evidences have demonstrated that association of miR-146a gene single nucleotide polymorphisms (SNPs) with risk of several diseases[2], but no genetic relevance studies of miR-146a gene polymorphisms to gout have been reported by now.

Objectives: To investigate the potential association of gout and the functional rs57095329 SNP of miR-146a in the Chinese Han population.

Methods: The rs57095329 SNP was detected in 448 primary gout patients (containing 76 tophi patients) and 418 healthy control subjects. Peripheral blood mononuclear cells (PBMCs) miR-146a expression was measured in 81 gout patients (including 32 tophi patients and 49 nontophi patients) and 47 healthy subjects.

Results: No significant difference was detected in the distribution of miR146 a rs57095329 between 448 gout patients and 418 healthy subjects $(P>0.05)$. However, significant differences were observed between 76 gout with tophi patients and 418 healthy subjects, between gout with tophi (76) and with no tophi patients (372) both in genotypes and allele distributions $(P<0.01$, respectively). Gout patients carrying $A G / G G$ genotypes had a 0.323 -fold reduced risk for tophi than those carrying AA genotype, and the $G$ allele carrier of gout patients had a 0.362 -fold reduced risk for tophi. Additionally, GG genotype was significantly associated with increased expression of miR-146a in 32 tophi patients (Figure).
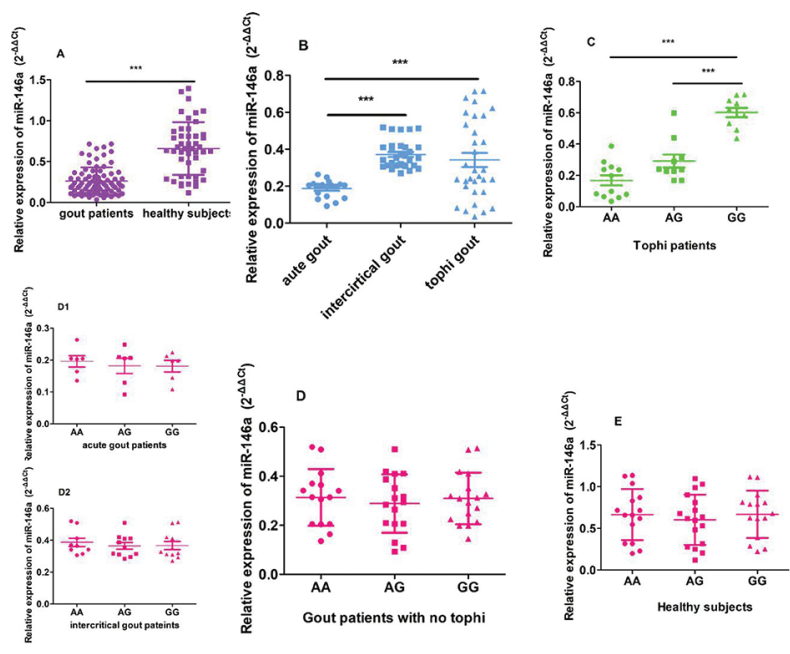

Conclusion: Our study shows a novel, significant association between the miR-146a rs57095329 polymorphism and a lower risk of tophi in gout patients. Furthermore, our findings suggest that this gene polymorphism might affect the genetic predisposition to tophi development and modulate the expression of miR-146a level in tophi patients. This new knowledge about miR-146a may be clinically important and confirms a role for miR$146 \mathrm{a}$ in the pathophysiology of tophi, with potentially important therapeutic implications.

\section{REFERENCES:}

[1] Zhang QB, Qing YF, Yin CC, Zhou L, Liu XS, Mi QS, et al. Mice with miR146a deficiency develop severe gouty arthritis via dysregulation of TRAF 6, IRAK 1 and NALP3 inflammasome. Arthritis Res Ther. 2018;20:45.

[2] Luo X, Yang W, Ye DQ, Cui H, Zhang Y, Hirankarn N, et al. A functional variant in microRNA-146a promoter modulates its expression and confers disease risk for systemic lupus erythematosus. PloS Genet. 2011;7: e1002128.

Disclosure of Interests: Yu-Feng Qing Grant/research support from: Sichuan Youth Science and Technology (2016JQ0053), and the Department of Science and Technology of Sichuan Province (2018JY0257), Ting Yi: None declared, Zi-Yi Tang: None declared, Jian-Xiong Zheng: None declared, Qin Xiong: None declared, Quan-Bo Zhang: None declared DOI: 10.1136/annrheumdis-2019-eular.3471

\section{FRI0019 URINARY TRANSCRIPTS AS BIOMARKERS OF LUPUS NEPHRITIS}

Natalie Rosenwasser ${ }^{1,2}$, Seunghee Hong ${ }^{2}$, Lynnette Walters ${ }^{3}$, Phuong Nguyen ${ }^{4}$, Cindy Wang ${ }^{1}$, Jeanine Baisch ${ }^{2}$, Peter Oxley ${ }^{2}$, Marina Ohouo ${ }^{2}$, Simone Caielli ${ }^{2}$, Cynthia Smitherman ${ }^{4}$, Yuanyuan Wang ${ }^{4}$, Tracey Wright ${ }^{5}$, Karen Onel ${ }^{1}$, Virginia Pascual' ${ }^{2}{ }^{1}$ Hospital for Special Surgery, New York, United States of America; ${ }^{2}$ Weill Cornell Medicine, New York, United States of America; ${ }^{3}$ Texas Scottish Rite Hospital, Dallas, United States of America; ${ }^{4}$ Baylor Scott and White, Dallas, United States of America; ${ }^{5}$ UT Southwestern Medical Center, Dallas, United States of America

Background: Children with systemic lupus erythematosus (cSLE) with kid ney involvement face considerable morbidity and mortality. Commonly used methods for assessing kidney involvement in cSLE, such as renal function tests, are non-specific and provide a limited picture of disease activity. Kidney biopsy, the mainstay to assess disease class and chronicity as well as response to treatment, is an invasive procedure. The field of transcriptomics has identified markers that have helped our understanding of cSLE pathogenesis.

Objectives: Transcriptional profiling of urinary cells has the potential to provide us with non-invasive biomarkers and has not been studied thus far. Our aim is to evaluate intracellular urinary transcripts in cSLE.

Methods: Using the Human Inflammation v2 panel on the nanoString nCounter platform, expression of 255 genes was profiled from 82 urinary pellets (all from 43 cSLE with differing classes of lupus nephritis).

Results: Healthy control samples yielded low quantities of RNA and were excluded from further analysis. The patient's raw transcript counts were normalized and the use of unsupervised learning analysis revealed robustness among classes with higher disease activity (higher SLE Disease Activity Index (SLEDAI) scoring). Statistical analysis (ANOVA) showed that Classes III or IV + V (mixed nephritis; highest average SLEDAI) display robust interferon and inflammasome-related signatures in comparison to other classes. Interestingly, samples from cSLE with no known nephritis also displayed an interferon signature, although less intense than the mixed class. Some transcripts such as C3 (complement 3) were uniquely dysregulated in the mesangial class. Therapeutically targetable pathways were identified in a number of patients (specifically in the mixed class). Conclusion: These studies are currently being extended in order to confirm the value of urinary transcriptional profiling as a source of biomarkers and to identify potential therapeutic targets in cSLE.

\section{REFERENCES}

[1] Pascual, et al., "Understanding Human Autoimmunity and Autoinflammation Through Transcriptomics," Annu Rev Immunol, 2017 Apr 26;35:337370. doi: 10.1146/annurev-immunol-051116-052225

[2] Bennett L, Palucka AK, Arce E, et al. Interferon and Granulopoiesis Signatures in Systemic Lupus Erythematosus Blood. The Journal of Experimental Medicine. 2003;197(6):711-723. doi:10.1084/jem.20021553.

[3] Banchereau R, Hong S, Cantarel B, Baldwin N, Baisch J, et al. 2016. Personalized immunomonitoring uncovers molecular networks that stratify lupus patients. Cell 165:551-65

[4] Chiche L, Jourde-Chiche N, Whalen E, Presnell S, Gersuk V, et al. 2014 Modular transcriptional repertoire analyses of adults with systemic lupus erythematosus reveal distinct type I and type II interferon signatures. Arthritis Rheumatol. 66:1583-95

[5] Brunner H.I., Bennett M.R., Gulati G., Abulaban K., Klein-Gitelman M.S., Ardoin S.P., Tucker L.B., Devarajan P. Urine biomarkers to predict response to lupus nephritis therapy in children and young adults (2017) Journal of Rheumatology, 44 (8), pp. 1239-1248.

Acknowledgement: Childhood Arthritis and Rheumatology Research Alliance 
Disclosure of Interests: None declared

DOI: 10.1136/annrheumdis-2019-eular.5241

\section{FRI0020 CORRELATION BETWEEN EXPRESSION LEVELS OF MIR-146A AND MIR-223 IN SYNOVIAL FLUID AND ULTRASOUND SCORES FOR ACTIVE SYNOVITIS IN RHEUMATOID ARTHRITIS PATIENTS}

Russka Shumnalieva ${ }^{1,1}$, Darina Kachakova ${ }^{2}$, Radka Kaneva ${ }^{2}$, Zlatimir Kolarov ${ }^{1}$, Simeon Monov ${ }^{1} .{ }^{1}$ Clinic of Rheumatology, Department of Internal Medicine, Medical University-Sofia, Sofia, Bulgaria; ${ }^{2}$ Molecular Medicine Center, Department of Medical Chemistry and Biochemistry, Medical University-Sofia, Sofia, Bulgaria

Background: MicroRNAs (miRNAs) are a class of small, non-coding RNAs that negatively regulate gene expression at posttranscriptional level. In the recent years studies have shown that in rheumatoid arthritis (RA) the systemic and local expression of certain miRNAs is altered [1-2]. The correlation between their expression levels and scores for disease activity and progression in RA make them possible candidate for biomarkers in the clinical practice.

Objectives: To analyze the expression levels of miR-146a and miR-223 in synovial fluid (SF) from RA patients in regard to the ultrasound scores for disease activity.

Methods: A total number of 48 RA patients according to the 1987 ACR criteria were included in the study. Expression levels of miR-146a and miR-223 SF were determined by qPCR (SybrGreen technology) and compared to healthy controls (HCs). Relative changes of gene expression levels of the studied miRNAs were calculated by $2^{-\Delta \triangle C t}$ method. Musculoskeletal ultrasound (MSUS) examination was performed by two independent examiners on ESAOTE, MyLab60 using both grey scale and power Doppler technic. A semi quantitative assessment of the peripheral joints was performed for detecting joint inflammation and determining the grade of synovial thickening and the degree of vascularization. Ultrasound features for active disease were correlated to the local expression of the studies miRNAs. SPSS was used for statistical analysis.

Results: RA SF showed overexpression of miR-146a (in 70.83\%, $\mathrm{p}=0.007$ ) and of miR-223 (in $79.17 \%, \mathrm{p}=1.64 \times 10-3$ ) when compared to HCs. There was a statistically significant correlation between the presence of synovitis and the degree of the power Doppler signal on MSUS and the local expression of miR-146a ( $p=0.030$ and $p=0.049$, respectively) and miR-223 $\left(p=6.19 \times 10^{-4}\right.$ and $p=0.003$, respectively). SF levels of miR-223 correlated also with the degree of synovial hypertrophy on MSUS ( $p=$ 0.013).

Conclusion: We found correlation between the SF expression of miR$146 \mathrm{a}$ and miR-223 and the ultrasound features of active joint inflammation. Further analysis with larger sets is needed to confirm if altered local miRNA expression could be used in the clinical practice as biomarker for disease activity especially in cases with subclinical synovitis.

\section{REFERENCES:}

[1] Li J, Wan Y, Guo Q, Zou L, Zhang J, Fang Y, et al. Altered microRNA expression profile with miR-146a upregulation in CD4+ $T$ cells from patients with rheumatoid arthritis. Arthritis Res Ther. 2010; 12(3):R81.

[2] Lu MC, Yu CL, Chen HC, Yu HC, Huang HB, Lai NS. Increased miR-223 expression in $\mathrm{T}$ cells from patients with rheumatoid arthritis leads to decreased insulin-like growth factor-1-mediated interleukin-10 production. Clin Exp Immunol. 2014; 177(3):641-51.

Acknowledgement: The study was supported by Grant 14-D/2012 and Grant 60/2013 funded by Medical University-Sofia

Disclosure of Interests: None declared

DOI: 10.1136/annrheumdis-2019-eular.6145

\section{Rheumatoid arthritis - comorbidity and clinical aspects}

\section{FRI0021}

ORAL HYGIENE STATUS IN RA PATIENTS AND PLACE OF RHEUMATOLOGIST IN THERAPEUTIC EDUCATION ON ORAL DENTAL HEALTH

saloua afilal ${ }^{1}$, Hanan Rkain ${ }^{1,2}$, Afaf Allaoui ${ }^{3}$, Oum Keltoum Ennibi ${ }^{3}$, Tahiri Latifa ${ }^{1}$, Nada Alami ${ }^{1}$, Fadoua Allali'. ${ }^{1}$ El Ayachi Hospital, Faculty of Medicine and Pharmacy of Rabat, Mohammed V University, Rheumatology B, rabat, Morocco: ${ }^{2}$ Laboratory of Physiology, Faculty of Medicine and Pharmacy of Rabat, Mohammed V University, rabat, Morocco; ${ }^{3}$ Periodontology department, Faculty of Dentistry, Mohammed V University, Rabat, Morocco, rabat, Morocco

Background: The relationship between oral hygiene and rheumatoid arthritis has been demonstrated by several studies.

Objectives: To evaluate oral hygiene status in rheumatoid arthritis (RA) patients.

To inform about the therapeutic education given by the rheumatologist on the importance of adequate oral hygiene in the management of RA

Methods: This is a cross-sectional study that included 100 consecutive RA patients ( $89 \%$ female, mean age 46.7 years, median disease duration of 8 years, mean specialized care duration of 3 years). A questionnaire evaluating oral hygiene status was administered. It focused on following items: the daily frequency of brushing, the modalities of brushing, the use of other means of oral hygiene, the regular follow-up at a dentist's doctor and the place of the rheumatologist in therapeutic education on the oral dental hygiene status.

Results: Table I illustrates the results of oral hygiene evaluation in RA patients.

Table I : Evaluation of Oral Hygiene in RA patients

\begin{tabular}{lc}
\hline Items & $\mathrm{N}=\mathbf{1 0 0}$ \\
\hline Daily Brushing Frequency (\%): & 18 \\
- 0 times/day & 37 \\
- once a day & 34 \\
- 2 times/day & 8 \\
- 3 times/day & 3 \\
- After each meal & \\
adequate brushing time ( $\geq 3 \mathrm{~min})(\%)$ & 31 \\
Correct brushing method (\%) & 14 \\
Use of other means of oral hygiene & 36 \\
(\%) & \\
Regular dentist visit (\%) & 2 \\
Never visit a dentist (\%) & 27 \\
\hline
\end{tabular}

Table II illustrates the results of the place of oral hygiene information in rheumato logic management.

Table II : Place of information on oral hygiene in rheumatologic care.

\begin{tabular}{lc}
\hline Questions & $\begin{array}{c}\text { Yes } \\
\text { (\%) }\end{array}$ \\
\hline $\begin{array}{lc}\text { 1. does your rheumatologist ever examined your oral cavity? } \\
\text { 2. have you ever been informed by your rheumatologist that poor oral hygiene }\end{array}$ & 6,0 \\
has a negative impact on your rheumatoid arthritis? & 11,0 \\
$\begin{array}{l}\text { 3. Does your rheumatologist ever recommended regular brushing of your } \\
\text { teeth? }\end{array}$ & 8,0 \\
$\begin{array}{l}\text { 4. Does your rheumatologist already recommended to you to consult a dentist? } \\
\end{array}$ & 10,0
\end{tabular}

Conclusion: This study illustrates the high prevalence of oral hygiene insufficiency in patients followed for RA. It also highlights poor therapeutic education given by the rheumatologist on the importance of adequate oral hygiene in the management of RA.

Disclosure of Interests: None declared

DOI: 10.1136/annrheumdis-2019-eular.7867 